\title{
Reply: The Effect of Primary Surgery in Patients with Stage IV Breast Cancer with Bone Metastasis Only (Protocol BOMET MF 14-01): A Multi-center, Registry Study
}

\author{
Atilla Soran, MD, MPH, FNCBC, FACS ${ }^{1}$, Efe Sezgin, PhD $^{2}$, Serdar Ozbas, MD ${ }^{3}$, Lutfi Dogan, MD $^{4}$, and \\ On Behalf of the Breast Health Working Group International
}

${ }^{1}$ Surgical Oncology, Magee-Women's Hospital, University of Pittsburg Medical Center, Pittsburgh, PA; ${ }^{2}$ Laboratory of Nutrigenomics and Epidemiology, Department of Food Engineering, Izmir Institute of Technology, Izmir, Turkey; ${ }^{3}$ Breast and Endocrine Surgeon, Ankara, Turkey; ${ }^{4}$ Department of Surgical Oncology, Ankara Oncology Training and Research Hospital, University of Health, Sciences, Ankara, Turkey

Dear Editor,

We'd like to thank Drs. Ishizuka and Horimoto for their letter to the editor. We're glad to see their interest to our prospective study. Regarding their recommendation that univariate Kaplan-Meier analysis does not eliminate confounding factors, such as age, tumor size, number of bone metastasis, etc., we employed propensity score methods for the results, including Fig. 1.

Statistical Analysis: A propensity score matching method is employed accounting for confounding covariates. Systemic therapy and surgery groups are matched based on significantly different clinicopathological factors, including age, tumor size, number of bone metastasis, histologic grade, treatment to primary breast tumor, and intervention to metastatic sites. Full matching gave the best matching and adequate balance (Supplemental Table 1;
Supplemental Fig. 1). Kaplan-Meier survival curves in systemic therapy and surgery groups are estimated in the matched sample. Stratified log-rank tests (stratifying on matched sets) used to compare survival curves. Cox regression models weighted by the matching weights and including subclasses as a cluster were run to estimate marginal hazard ratios (HR) and 95\% confidence intervals (CI). Statistical analyses were conducted with $\mathrm{R}$ version 4.1.0 (R Foundation for Statistical Computing, Vienna, Austria).

We believe that as the author stated in the letter these new analyses deliver more precise information to the readers.This propensity score matching method supports with an additional evidence that primary breast surgery should be in the treatment scheme in patients with de novo stage IV breast cancer.
Supplementary Information The online version contains supplementary material available at https://doi.org/10.1245/s10434021-10396-1.

(C) Society of Surgical Oncology 2021

First Received: 22 June 2021

Accepted: 22 June 2021;

Published Online: 7 July 2021

A. Soran

e-mail: asoran@upmc.edu 
FIG. 1 Combined

$(\mathrm{ST}+$ Surgery and Surgery + ST as a surgery group) survival analysis. $S T$ systemic treatment
BOMET Overall Survival $(\mathrm{N}=505)$

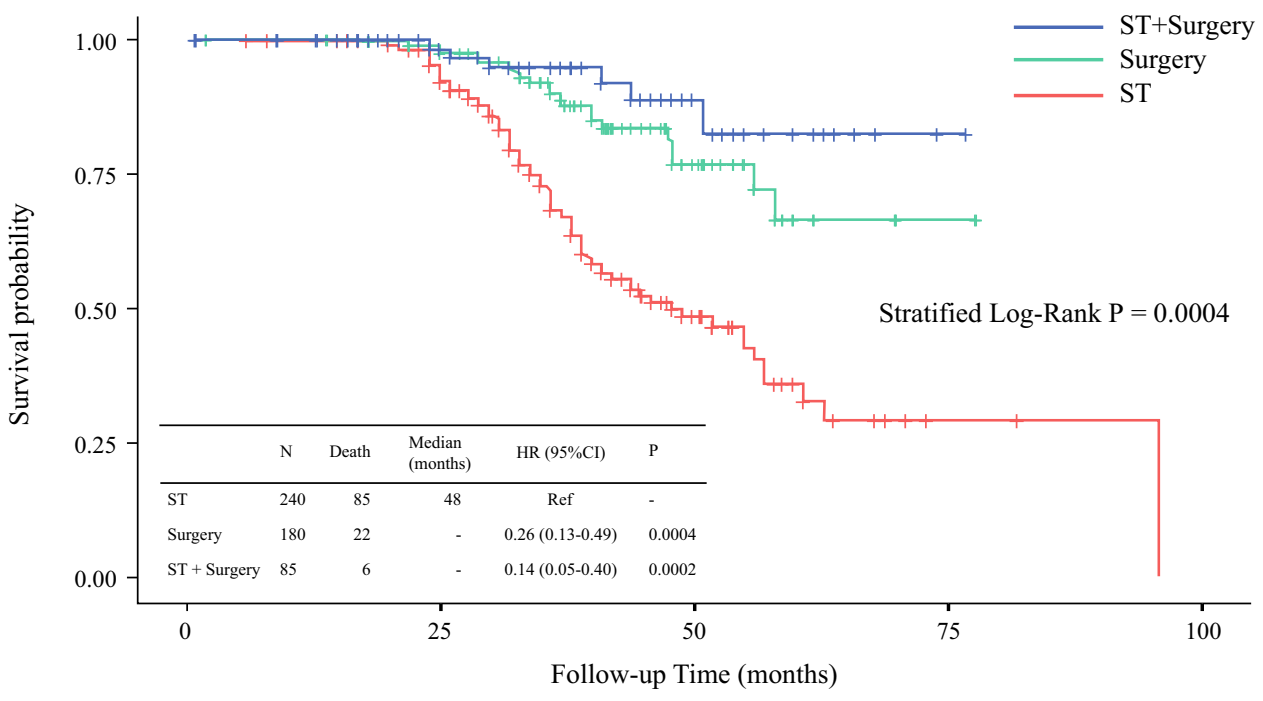

Surgery (Combined data) vs ST

BOMET Overall Survival (N=505)

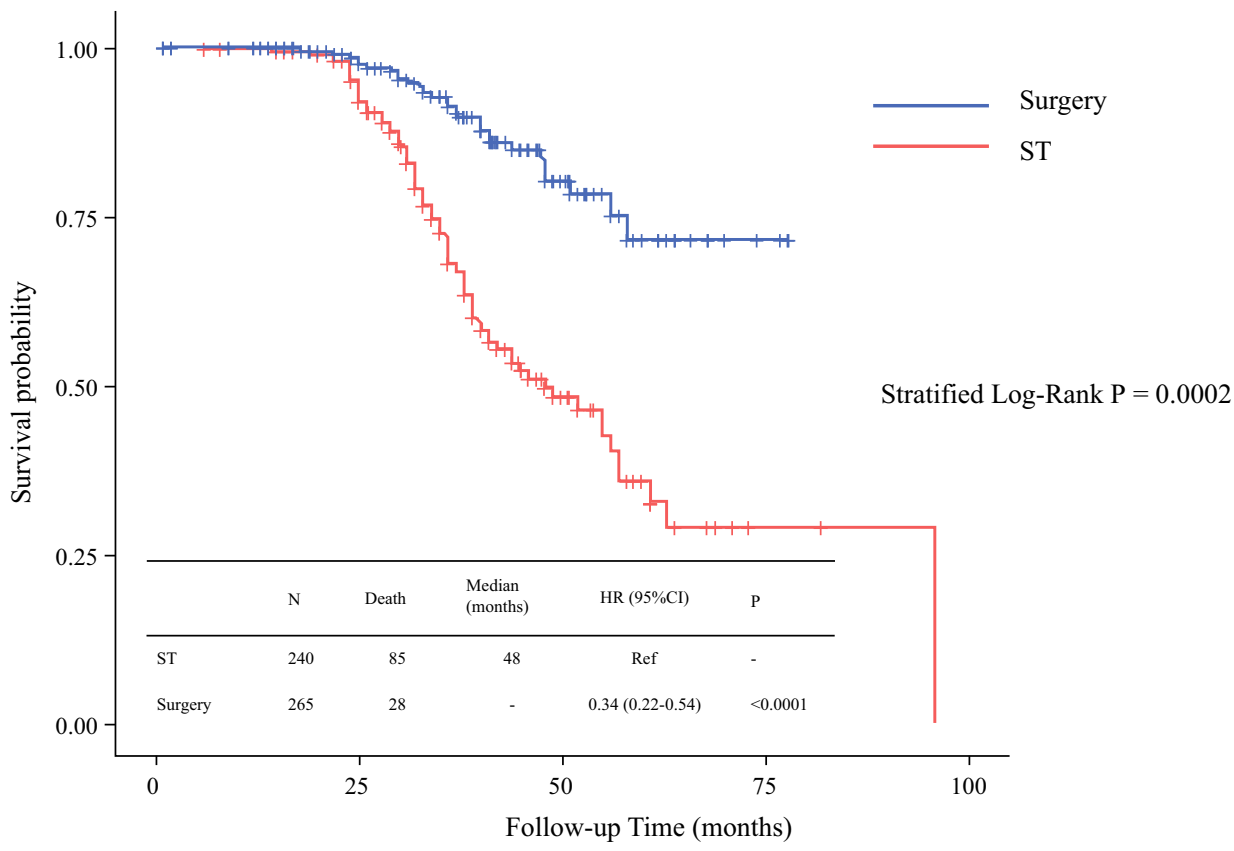

Publisher's Note Springer Nature remains neutral with regard to jurisdictional claims in published maps and institutional affiliations. 